\title{
SIFONAPTEROFAUNA DE TRES ESPECIES DE ROEDORES DE CONCEPCION, VIII REGION-CHILE
}

\author{
FLEAS (INSECTA: SIPHONAPTERA) ON THREE SPECIES OF RODENT AT \\ CONCEPCION, VIII REGION-CHILE
}

\author{
Mauricio E. Alarcón \\ Programa de Magíster en Ciencias mención Zoología. Universidad de Concepción. Depto. Zoología. Casilla 160-C. \\ Concepción. Chile. Email: mauralar@yahoo.com
}

\begin{abstract}
RESUMEN
Se estudió la sifonapterofauna recolectada en los roedores Abrothrix longipilis (Waterhouse), Abrothrix olivaceus (Waterhouse) y Rattus rattus Lineo, durante el período de mayo 2000-febrero 2001. Se informan aspectos taxonómicos y ecológicos que contribuyen al conocimiento de la fauna de sifonápteros de la VIII Región de Chile. Se obtuvo un total de 533 pulgas. Los resultados indican a $R$. rattus como nuevo hospedero para las pulgas Ctenoparia inopinata Rothschild, Sphinctopsylla ares (Rothschild) y Tetrapsyllus rhombus Smit en Chile y, a Neotyphloceras crassispina Rothschild como un nuevo registro para la Región. Plocopsylla wolfssohni (Rothschild) fue la especie más abundante en invierno y Ctenoparia inopinata en primavera.
\end{abstract}

Palabras Claves: Pulgas, VIII Región, nuevo hospedero, nuevo registro, roedores.

\section{ABSTRACT}

Siphonapterofauna occurring on Abrothrix longipilis (Waterhouse), Abrothrix olivaceus (Waterhouse) and Rattus rattus Lineo, collected between may 2000 and february 2001 was studied. Taxonomic and ecological background about 533 fleas are given. $R$. rattus is recorded as a new host in Chile for Ctenoparia inopinata Rothschild, Sphinctopsylla ares (Rothschild) and Tetrapsyllus rhombus Smit. The first record for the species Neotyphloceras crassispina Rothschild is informed for the VIII Región. Plocopsylla wolffsohni (Rothschild) appears as the more abundant species during winter time. Ctenoparia inopinata is the more abundant species in spring time.

KeYwords: Fleas, VIII Region, new host, new record, rodents.

\section{INTRODUCCION}

Los primeros antecedentes sobre la fauna de sifonápteros presentes en Chile fueron reportados por Blanchard (1852). Sin embargo, es sólo a principios de 1900 cuando se describen las primeras seis especies de sifonápteros chilenos (Rothschild, 1904, 1906, 1909a, 1909b y 1910). A partir de esa fecha y con motivo de los brotes de peste bubónica y tifus murino en Sudamérica entre los años 19201950, las investigaciones se enfocan desde una perspectiva epidemiológica hacia la identificación de los agentes etiológicos y sus vectores biológicos las pulgas (Ruiz del Río 1938; Macchiavello 1948, 1954).

Una vez desaparecidos los brotes de peste bubónica en la región (incluido Chile), paulatinamente se fue abandonando el estudio del 
grupo. Sólo taxónomos extranjeros en asociación con mastozoólogos nacionales han continuado trabajando el material obtenido en esporádicas recolecciones en terreno o de la recepción de especímenes para identificación, lo que ha dado como resultado en los últimos 30 años la descripción de 30 especies chilenas nuevas para la ciencia ( Beaucournu \& Gallardo 19781989 y 1991b, Beaucournu \& Kelt 1990; Beaucournu \& TorresMura 1986; Beaucournu et al. 1986; Beaucournu et al. 1988; Hastriter 2001; Jameson \& Fulk 1977; Lewis \& Spotorno 1984 y Smit \& Rosicky 1972). Varias de estas especies fueron recolectadas en roedores por Beaucournu \& Gallardo, (1991 a; 1992) cuya información se encuentra sintetizada en el "Catalogue Provisoire des Puces du Chili (Insecta; Siphonaptera).

Los roedores del género Abrothrix Waterhouse y en especial las especies longipilis y olivaceus son muy abundantes en el territorio chileno (Murúa et al. 1987), siendo capturadas con mucha frecuencia, lo que los ha llevado a convertirse en proveedores de valiosa información para el estudio de las pulgas. Sin embargo, esta información es incompleta y deriva de recolecciones en diferentes épocas del año y lugares que no permiten relacionar los hallazgos con las características propias de los hospederos, ni tampoco caracterizar especificidad ni estacionalidad para el caso de las pulgas (Hastriter et al.2001). Por otro lado, Rattus rattus y sus pulgas asociadas siempre han estado relacionados como vectores de enfermedades ( $\mathrm{M}^{\mathrm{C}}$ Evedy 1988). Sin embargo, los registros de esta relación para la región no están claros y datan de mediados del siglo pasado (Ruiz del Río 1938).

Teniendo en consideración que los roedores han sido el principal sustrato de investigación en Chile para éstos insectos, proporcionando en su mayor parte la información taxonómica disponible sobre sifonápteros, lo que ha dado origen a catálogos y descripciones de especies (Smit 1968; Alarcón 2000), y que aspectos ecológicos de las pulgas como por ejemplo su fenología y especificidad son pobremente entendidos, es que este artículo pretende contribuir al escaso conocimiento de las pulgas, en Chile. Se discute además a Rattus rattus como un nuevo hospedero para 3 especies de pulgas Ctenoparia inopinata, Sphinctopsylla ares y Tetrapsyllus rhombus y la presencia de Neotyphloceras crassispina como un nuevo registro para la VIII Región.

\section{MATERIALES Y METODOS}

Las 3 especies de roedores estudiados, Abrothrix longipilis, Abrothrix olivaceus y Rattus rattus, fueron recolectadas vivas mediante el uso de trampas Shermann cebadas con avena en El Cerro La Gallina, Barrio Universitario, VIII Región, Chile $\left(36^{\circ} 43 \mathrm{~S}\right.$ y $\left.73^{\circ} 12 \mathrm{~W}\right)$. El área corresponde a un paisaje intervenido, en el que predomina vegetación arbórea exótica (Eucalyptus globulus (Labille), Pinus radiata Don, con fragmentos de vegetación nativa secundaria). Una vez capturados los roedores fueron colocados en una bolsa plástica y sacrificados in situ por dislocación cervical. Se realizó un total de 20 muestreos con frecuencia quincenal, entre mayo de 2000 a febrero de 2001. Para ello se colocó en forma paralela separadas a 15 metros, 2 grillas de 25 trampas dispuestas cada 5 metros, por 2 noches consecutivas, siguiendo la naturaleza del terreno y tratando de abarcar la mayor parte de los biotopos presentes (Beaucournu \& Launay 1990).

En el laboratorio, se procedió a abrir las bolsas y recuperar las pulgas. Primero se concentró la atención en aquellos especímenes que habían abandonado el cuerpo del animal. El resto fue extraído con la ayuda de un peine fino a favor y en contra del pelo, asegurando un éxito de $100 \%$ en la recolecta. Cada roedor fue medido, sexado, diferenciado según grupo etario en adultos y juveniles (Yáñez \& Jaksic 1977; Pearson 1983) e individualizado con un número de registro para ser ingresado a las Colecciónes Científicas de la Universidad de Concepción (UCCC). Los parásitos fueron fijados en alcohol $70^{\circ}$, y guardados en frascos para su posterior identificación.

Las pulgas fueron tratadas según la técnica de Hastriter: aclaradas en $\mathrm{KOH} 20 \%$ por 24 horas a temperatura ambiente, deshidratadas en concentraciones crecientes de alcohol, diafanizadas en creosota y finalmente, montadas en bálsamo de Cánada.

Para la identificación taxonómica de los roedores se usaron las claves de Osgood (1943), Mann (1978), Muñoz-Pedreros (2000), además de la comparación con ejemplares pertenecientes a las Colecciones Científicas de Referencia de la Universidad de Concepción (UCCC). Para el caso de las pulgas, éstas se identificaron según las claves 
de Hopkins \& Rothschild (1956, 1962 y 1966); Smit (1973); Dunnet \& Mardon (1974 / 1991) y la cooperación de los especialistas J.C Beaucournu (Universidad de Rennes-Francia) y Michael W. Hastriter (Brigham Young University-USA). Para el ordenamiento taxonómico de las especies de pulgas estudiadas se sigue a Dunnet \& Mardon, (1991).

\section{RESULTADOS}

Durante las actividades en terreno fueron recolectados un total de 135 roedores que correspondieron a las especies Abrothrix olivaceus: 89 ejemplares; Abrothrix longipilis: 40 ejemplares y 6 ejemplares de Rattus rattus. En ellos se detectó la presencia de 533 pulgas, 227 de las cuales se recolectaron en $A$. olivaceus, 290 en $A$. longipilis y 16 en Rattus rattus.

\section{LISTADO DE ESPECIES}

\section{FAMILIA CERATOPHYLLIDAE DAMPF, 1908}

\section{Nosopsyllus fasciatus (Bosc 1831)}

Material examinado: $2 \mathrm{~h}$ ( + ): $1 \mathrm{~h}$, vi.2000. Chile: VIII Región-Concepción, Barrio UniversitarioCerro la Gallina; M. Alarcón \& M. Daza col.; 1h, viii.2000, misma localidad. M. Alarcón col. (Ex. A. olivaceus).

Distribución CONOCIDA: “Cosmopolita esencialmente en zonas urbanas, normalmente fuera de las zonas temperadas. En Chile: Elqui (Coquimbo), Santiago, Valparaíso; Costa de Chile y hacia el sur de Concepción; Bío-Bío" (Beaucournu \& Gallardo 1991a).

HosPederos CONOCIDOS: "Abrothrix olivaceus, Homo sapiens Lineo, Marmosa elegans (Waterhouse), Rattus spp" (op.cit.).

Observaciones: Los miembros de la Familia Cerathophyllidae tienen una distribución holártica en aves y mamíferos. El género Nosopsyllus tiene actualmente 61 especies distribuidas en las regiones Paleártica, Etiópica y Oriental. N. fasciatus (Bosc) y N. londiniensis (Rothschild 1903) son cosmo-politas y, se encuentran en ratas y lauchas (Dunnet \& Mardon 1991). Ruiz del Río 1938 y Macchiavello 1948 la encuentran con mucha frecuencia en Concepción en roedores domésticos. A partir de este trabajo se determinó la presencia de $N$. fasciatus en A. olivaceus, lo que probablemente estaría indicando que este roedor representaría un recurso alternativo, por lo que podría considerarse una infestación accidental.

Los 2 especímenes hembra fueron recolectados en invierno. Tal vez el clima y las condiciones térmicas de la región estarían limitando la proliferación de $N$. fasciatus durante los meses de primavera y verano.

\section{FAMILIA CTENOPHTHALMIDAE ROTHSCHILD, 1915}

\section{Chiliopsylla allophyla (Rothschild 1908).}

Material examinado: $10^{\star}$ y $19: 10^{\star}$, x.2000. Chile: VIII Región-Concepción, Barrio UniversitarioCerro la Gallina; M. Alarcón \& M. Daza col. y, 1 ㅇ xi.2000, misma localidad. M. Alarcón col. ( Ex. A. olivaceus).

Distribucion conocida: "Argentina; Chile, Cautín (tipos); Valdivia, Osorno, Chiloé y Concepción" (Beaucournu \& Gallardo, 1991a y Hastriter et al. 2001).

Hospederos conocidos: "Abrothrix longipilis, $A$. o. olivaceus, A. olivaceus brachiotis (Waterhouse), A. sanborni (Osgood), Aconaemys porteri Thomas, Auliscomys micropus (Waterhouse), Dromiciops australis (Philippi) e Irenomys tarsalis (Philippi)" (op. cit.).

OBSERVACIONEs: Beaucournu \& Gallardo, (1991a) y Autino \& Lareschi (1998) han encontrado a esta especie, aunque en bajo número, parasitando roedores múridos y cávidos tanto en Argentina como en Chile.

Sin embargo Hopkins \& Rothschild 
(1966) la describieron en Dromiciops australis (Philippi, 1893) (Marsupialia: Microbiotherinae) con altas intensidades de infestación, sugiriendo un posible caso de concordancia entre hospedero-parásito. Lo anterior ha sido corroborado por Hastriter $e t$ al. (2001), quien define como su hospedero natural a este pequeño marsupial, coincidiendo estrechamente con sus rangos distribucionales.

El hallazgo de Chiliopsylla allophyla en Abrothrix olivaceus permite sugerir la presencia de Dromiciops gliroides (Philippi, 1893) en los bosques del "Cerro Caracol" en Concepción y que Chiliopsylla allophylla utilizaría Abrothrix olivaceus como hospedero alternativo dada la baja densidad poblacional de D. gliroides (Glade 1988). Lo anterior estaría sustentado por el hallazgo primaveral de esta especie de pulga, que coincide con el comportamiento sexual de este marsupial (Muñoz- Pedreros 2000).

\section{Neotyphloceras crassispina Rothschild} 1914

Material examinado: $14 \sigma^{*}$ y $16 \%: 10$, v. 2000. Chile: VIII Región-Concepción, Barrio Universitario-Cerro la Gallina; M. Alarcón \& P. Chandía col.; $3 \mathrm{~mm}$, vi.2000, misma localidad. M. Alarcón col.; 1 ơ, 1 h, vii.2000, misma localidad. M. Alarcón \& C. Alarcón col.; $4 \sigma^{\star}, 4$ ㅇ, viii.2000, misma localidad. M. Alarcón \& M. Daza col.; $30^{\star}, 6$ ㅇ, ix.2000, misma localidad. M. Alarcón col.; $10^{\star}, 4$ 우, x.2000, misma localidad. M. Alarcón col.; $10^{\top}$, 1 + , ii.2001, misma localidad. M. Alarcón col. ( Ex. A. olivaceus).

$310^{\star}$ y 45 우 $10^{\top}, 1$ ㅇ, v. 2000 . Chile: VIII Región-Concepción, Barrio UniversitarioCerro la Gallina; M. Alarcón col.; $60^{\text {* }}$, vi.2000, misma localidad. M. Alarcón col.; $3 o^{\star}, 2$ \% , viii.2000, misma localidad. Alarcón col.; $7 \sigma^{\star}, 13$ \% , ix.2000, misma localidad. M. Alarcón col.; $30^{\star}, 3$ ㅇ, x.2000, misma localidad. M. Alarcón col.; $40^{\star}, 18$ \% , xi.2000, misma localidad. M. Alarcón col.; $10^{\star}, 3$ 우, xii.2000, misma localidad. M. Alarcón \& M.
Daza col.; $50^{\star}, 5$ , i. 2000, misma localidad. M. Alarcón col.; $10^{\star}$, i.2001, misma localidad.M. Alarcón col. (Ex. A. longipilis ).

$30^{\text {t }}$ y 4 ㅇ: $1 \mathrm{~h}$, vi.2000, misma localidad. M. Alarcón col. $1 \sigma^{*}, 1$ 우, viii.2000, misma localidad. M. Alarcón col. $10^{\star}, 2$ ㅇ, i.2001, misma localidad. M. Alarcón col. $10^{\star}$, ii.2001, misma localidad. M. Alarcón col. (Ex. R. rattus).

Distribucion Conocida: "Ecuador, Perú, Bolivia, Argentina (Smit 1955) y Chile: Aconcagua, Curicó, Valparaíso y Santiago" ( Beaucournu \& Gallardo 1991a). Concepción (presente trabajo).

Hospederos conocidos: "Abrothrix spp, Chinchi-llula spp, Olygoryzomys spp, Octodon spp y Phyllotis spp." (op. cit.).

ObSERVACiOnes: Dos especies son conocidas del género Neotyphloceras Rothschild 1914: N. rosenbergi (Rothschild 1904) descrita para Ecuador y Perú (Hopkins \& Rothschild 1966) y $N$. crassispina Rothschild 1914, con tres subespecies: crassispina crassispina Rothschild 1914 en Ecuador, Perú, Bolivia, Argentina y Chile; crassispina chilensis Jordan, 1936 en Perú, Argentina y Chile y crassispina hemisus Jordan 1936, en Perú, Bolivia, Argentina y Chile (Beau-cournu \& Gallardo 1991b).

El hallazgo de Neotyphloceras crassispina crassispina Rothschild, en los Bosques del Barrio Universitario (VIII Región-Chile), amplía considerablemente la distribución geográfica de esta población hacia el sur (Smit 1968), de modo que lo sugerido por Smit (1968) en el sentido de la existencia de 2 dos subespecies ( $N$. crassispina crassispina y crassispina chilensis) coexistiendo en simpatría en la provincia de Valparaíso, no es sustentable bajo el concepto biológico de población (Begon et al. 1996).

Smit en 1968 sostiene que $N$. crassispina crassispina es una subespecie común en roedores cricétidos de los géneros Abrothrix, Oryzomys, Phyllotis y Chinchillula. Sobre la base de nuestros resultados se determinó que $N$. crassispina crassispina prefiere como hospedero a A. longipilis sobre todo en las épocas de invierno y primavera. 
Gayana 67(1), 2003

\section{FAMILIA HYSTRICHOPSYLLIDAE TIRABOSCHI, 1904}

\section{Ctenoparia inopinata Rothschild 1911}

Material EXAMinado: $300^{\star}$ y $43 q: 20^{\star}$, vi.2000.Chile: VIII Región-Concepción, Barrio UniversitarioCerro la Gallina; M. Alarcón col. ; $3 \sigma^{*}, 1$ ㅇ, vii.2000, misma localidad. M. Alarcón col. ; $20^{\star}$, viii.2000, misma localidad. M. Alarcón col. ; $10^{\circ}$, ix.2000, misma localidad. M. Alarcón \& M. Daza col. ; $3 \sigma^{\star}, 21$ ㅇ , x.2000, misma localidad. M. Alarcón col. ; $1 \sigma^{\star}, 1$ ㅇ , xi.2000, misma localidad. M. Alarcón col. ; $60^{\star}, 8$ \% , xii. 2000 , misma localidad. M. Alarcón col. ; $30^{\circ}, 1$ , i.2001, misma localidad. M. Alarcón col. ; $90^{\star}, 11$ † , ii.2001, misma localidad. M. Alarcón col. ( Ex. A. olivaceus).

$410^{\top}$ y 68 \% : $10^{\star}, 2$, vi.2000. Misma localidad. M. Alarcón \& P. Chandía col.; $10^{\star}, 1$ \%, viii.2000, misma localidad. M. Alarcón col.; $40^{\star}, 9$ ㅇ, ix.2000, misma localidad. M. Alarcón col.; $110^{\star}$, 16 , x.2000, misma localidad. M. Alarcón col.; $90^{*}, 20$ ㅇ x xi.2000, misma localidad.M. Alarcón col.; $80^{*}, 6$ ㅇ, xii.2000, misma localidad. M. Alarcón col.; 40 $12 \%$, i.2001, misma localidad. M. Alarcón col.; $3 \sigma^{\star}, 2$ ㅇ, ii.2001, misma localidad. M. Alarcón col. (Ex. A. longipilis). 1 ㅇ: 1 ㅇ, viii.2000, misma localidad. M. Alarcón col. (Ex. R. rattus).

Distribución CONOCida: "Argentina, Chile: Elqui (Coquimbo), Valparaíso, Santiago, Concepción, Malleco, Cautín, Valdivia, Osorno, Chiloé, Coyhaique (Aisén).

HoSPEDEROS CONOCIDOS: "Abrothrix longipilis; A. longipilis apta (Oosgod); A. o. olivaceus; A. olivaceus brachiotis; Abrothrix sanborni, Aconaemys porteri, Geoxus valdivianus (Philippi) y Oligoryzomys longicaudatus (Bennet) "(fide Beaucournu \& Gallardo 1991a). Rattus rattus (presente trabajo).

ObSeRvaCiones: A pesar de la insuficiencia de las prospecciones, se ha especulado para el caso de Ctenoparia inopinata su preferencia por A. olivaceus y A. longipilis (Jameson \& Fulk 1977). Hastriter et al. (2001) comparten esta opinión, pero se inclinan por proponer a A. longipilis como el hospedero más seleccionado por $C$. inopinata y agrega que esta especie es común en roedores que viven en el bosque templado lluvioso de Argentina pero es infrecuente en similares condiciones en Chile.

La mayor parte de los hallazgos de $C$. inopinata la relacionan a hospederos del género Abrothrix. De hecho Rothschild en 1910 describe como holotipo a una hembra procedente de un individuo de A. olivaceus, el macho descrito por Beaucournu et al. (1986) también procede de un ejemplar de esta misma especie. De los 23 ejemplares de $C$. inopinata recolectados a la fecha (Beaucournu \& Kelt 1990; Beaucournu et al. 1986; Hastriter et al. 2001 y Rothschild 1910), 13 han sido capturados en A. olivaceus y 5 en $A$. longipilis.

Los resultados aquí presentados con un total de 73 individuos (30 o y 43 ㅇ) C. inopinata capturados en A. olivaceus y 109 (41 o y 68 \% ) en $A$. longipilis, corresponden al mayor número de especímenes de esta especie registrados para Chile, ya que Beaucournu \& Alcover (1989) recolectaron en la provincia de NeuquénArgentina 101 ejemplares (49 $0^{\star}$ y 52 \%), sobre 99 especímenes de A. longipilis.

En base a estos antecedentes se concluye que $C$. inopinata tiende a encontrarse con mayor frecuencia en la estación de primavera, siendo su hospedero más seleccionado A. longipilis. Esto confirmaría la propuesta de Hastriter et al. (2001) en relación a una posible especificidad y preferencia de $C$. inopinata por A. longipilis.

\section{FAMILIA RHOPALOPSYLLIDAE OUDEMANS, 1909}

\section{Tetrapsyllus rhombus Smit 1955}

Material examinado: $90^{\star}$ y $69: 2 \mathrm{~m}$, v. 2000. Chile: VIII Región-Concepción, Barrio Universitario-Cerro la Gallina; M. Alarcón \& M. Neira col.; $20^{\star}, 1$ 우, vi.2000, misma localidad. M. Alarcón col.; $3 \sigma^{\star}, 2$ 우, viii.2000, misma localidad. M. Alarcón col.; $20^{\star}, 2$, ix.2000, misma localidad. M. Alarcón col.; 1 \% , i.2001, misma localidad. M. Alarcón col. (Ex. A. olivaceus). $150^{\star}$ y 10 우: 2 우, vi.2000, misma localidad. M. Alarcón \& M. Daza col.; $10^{\star}$, viii.2000, misma localidad. M. Alarcón col.; 1 우, ix.2000, misma 
localidad. M. Alarcón col.; $6 \overbrace{}^{\star}, 4$ † , x.2000, misma localidad. M. Alarcón col.; $40^{\star}, 2$ \% , xi.2000, misma localidad. M. Alarcón col.; $1 \overbrace{}^{\star}, 1$ ค , xii.2000, misma localidad. M. Alarcón col.; $3 \sigma^{\star}$, i.2001. (Ex. A. longipilis).

2울 1 우, vi.2000, misma localidad. M. Alarcón $\&$ M. Daza col. 1 ㅇ, viii.2000, misma localidad. M. Alarcón col. (Ex. R. rattus).

Distribución CONOCiDA: "Argentina; Chile: Elqui (Coquimbo), Malleco, Cautín (paratipo), Concepción, Chilóe, Valdivia, Osorno, Coyhaique (Aisén) y General Carrera (Aisén)" (Beaucournu \& Gallardo 1992).

Hospederos Conocidos: "Abrothrix longipilis; A. o. olivaceus, A. olivaceus brachiotis, Aconaemys porteri, Auliscomys micropus, Ctenomys maulinus Philippi, Euneomys mordax Thomas, Geoxus valdivianus, Rattus norvergicus Berkenhout y Reithrodon physodes (Olfers)" (op.cit. ). Rattus rattus (presente trabajo).

Tetrapsyllus tantillus (Jordan \& Rothschild 1923)

Material examinado: $60^{\circ}$ y $8 \%: 2 \mathrm{~m}, \mathrm{v} .2000$. Chile: VIII Región-Concepción, Barrio Universitario-Cerro la Gallina; M. Alarcón col.; 2 \%, viii.2000, misma localidad. M. Alarcón col.; 2 \%, x.2000, misma localidad. M. Alarcón \& M. Daza col.; $20^{\star}, 2+$, xii.2000, misma localidad. M. Alarcón col.; 1 우 i.2001, misma localidad. M. Alarcón col.; $2 \sigma^{\star}, 1$ 우 , ii.2001, misma localidad. M. Alarcón col. (Ex. A. olivaceus).

$30^{\star}$, xi.2000, misma localidad. M. Alarcón col. (Ex. A. longipilis).

Distribución CONOCIDA: "Argentina ; Chile: Elqui (Coquimbo), Limarí, Valparaíso, Santiago, Malleco, Bío-Bío, Coyhaique (Aisén), General Carrera (Aisén), Magallanes y Ultima Esperanza" (Beaucournu \& Gallardo 1992)

HoSPEDEROS CONOCIDOS: "Abrothrix longipilis (Waterhouse), A. o. olivaceus (Waterhouse); Abrothrix xanthorhinus (Waterhouse), Auliscomys micropus (Waterhouse), Ctenomys magellanicus Bennett, Euneomys mordax Thomas,
Marmosa elegans (Waterhouse), Phyllotis darwini (Waterhouse), P. xantophygus (Waterhouse), Reithrodon physodes (Olfers) y Spalacopus cyanus (Molina)" (op. cit.).

Observaciones: Dos especies de la familia Rhopalopsyllidae, Tetrapsyllus rhombus y $T$. tantillus, fueron recolectadas durante la investigación. Dada la escasez de registros poco se sabe acerca de estas 2 espcies. Sin embargo, los datos existentes en la literatura para $T$. rhombus apuntan hacia pequeños roedores como A. olivaceus, A. longipilis y Oligoryzomys longicaudatus (Bennett), aunque esto sólo puede reflejar el hecho de que estos roedores son los más comúnmente capturados mediante el uso de trampas Shermann.

\section{FAMILIA STEPHANOCIRCIDAE WAGNER, 1928}

Plocopsylla wolffsohni (Rothschild 1909)

Material examinado: $90^{*}$ y $12 \%: 10^{*}, 5$ 우 vi.2000. Chile: VIII Región-Concepción, Barrio Universitario-Cerro la Gallina; M. Alarcón col.; $20^{\star}, 5$ ㅇ, vii.2000, misma localidad. M. Alarcón col.; $20^{\star}, 2+$, viii.2000, misma localidad. M. Alarcón col.; $4{ }^{\star}$, ix.2000, misma localidad. M. Alarcón col.

(Ex. A. olivaceus).

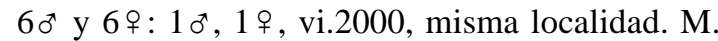
Alarcón col.; $20^{\star}, 2$, , viii.2000, misma localidad. M. Alarcón col.; $10^{\star}, 3$ ㅇ, ix.2000, misma localidad. M. Alarcón col.; $20^{\star}$, x.2000, misma localidad. M. Alarcón col. (Ex. A. longipilis).

DistRibuCión CONOCIDA: "Argentina; Chile: Elqui (Coquimbo), Valparaíso, Santiago, Nuble y Concepción" (Beaucournu \& Gallardo 1991a).

Hospederos conocidos: "Abrothrix lon-gipilis, A. o. olivaceus, Chelemys macronyx (Thomas), Octodon degus (Molina) y Phyllotis darwini (Waterhouse)" (op. cit.).

ObSeRVACIONEs: Esta especie se ha descrito en muchos roedores, principalmente en cricétidos (múridos sensu Muñoz-Pedreros, 2000), tanto de 
Argentina como de Chile (Jameson \& Fulk, 1977). $P$. wolfsohni fue recolectada tanto de $A$. olivaceus como de A. longipilis, con un mayor número de individuos capturados en el período otoño-invierno.

\section{Sphinctopsylla ares (Rothschild 1911)}

Material examinado: $180^{*}$ y 52 우 $3 \mathrm{~m}, 5$ 우, v.2000. Chile: VIII Región-Concepción, Barrio Univer-sitario-Cerro la Gallina; M. Alarcón col.; $5 o^{\star}, 9$ ㅇ, vi.2000, misma localidad. M. Alarcón col.; $50^{\star}, 8$ 우, vii.2000, misma localidad. M. Alarcón col.; 1 ơ $^{\star} 12$ ㅇ, viii.2000, misma localidad. M. Alarcón col.; $20^{\star}, 4$ + , ix.2000. misma localidad. M. Alarcón col.; 9 ㅇ, x.2000, misma localidad. M. Alarcón col.; 2 ㅇ, xii.2000, misma localidad. M. Alarcón col.; $20^{\star}, 3$ 우, ii.2001, misma localidad. M. Alarcón col. (Ex. A. olivaceus).

$21 \sigma^{\star}$ y 44 ㅇ: $10^{\star}, 2$ ㅇ, v.2000, misma localidad. M. Alarcón col.; $1 \sigma^{*}, 2$ \% , vi.2000, misma localidad. M. Alarcón col.; $10^{\star}, 4$ 우, viii.2000, misma localidad. M. Alarcón \& M. Daza col.; $60^{*}$, 4 ㅇ, ix.2000, misma localidad. M. Alarcón col.; $3 o^{\star}, 9$ ㅇ, x.2000, misma localidad. M. Alarcón col.; $60^{\star}, 12$ o , xi.2000, misma localidad. M. Alarcón col.; 2 ㅇ, xii.2000, misma localidad. M. Alarcón col.; $3 \sigma^{\star}, 9$ ㅇ, i.2001, misma localidad. M. Alarcón col. ( Ex. A. longipilis ).

6오: 2 우, vi.2000, misma localidad. M. Alarcón col. 4 ㅇ, viii.2000, misma localidad. M. Alarcón col. (Ex. R. rattus).

Distribución CONOCIDA: “Argentina; Chile: Elqui (Coquimbo), Limarí, Valparaíso, Nuble (Chillán), Concepción, Malleco, Cautín, Valdivia, Osorno, Chiloé, Coyhaique (Aysén), General Carrera (Aisén) y Magallanes. (Beaucournu \& Gallardo 1991a)".

HosPederos CONOCIDOS: "Abrothrix longipilis; A. o. olivaceus, Abrothrix xanthorhinus (Waterhouse), Aconaemys porteri, Auliscomys micropus, Chelemys macronyx, Euneomys mordax, Geoxus valdivianus, Marmosa elegans (Waterhouse), Orlygoryzomys longicaudatus, Phyllotis darwini, P. xanthopygus (Waterhouse), Reithrodon physodes y Spalacopus cyanus
(Molina)" (op. cit.). Rattus rattus (presente trabajo).

Observaciones: A diferencia de $C$. inopinata, tanto Sphinctopsylla ares como Tetrapsyllus rhombus, han sido calificadas como especies eurixénicas. Al respecto Beaucournu \& Kelt (1990) al revisar 700 pulgas extraídas de roedores cricétidos y octodóntidos, concluyeron que $S$. ares fue la especie más abundante entre los miembros recolectados de la familia Stephanocircidae.

En relación a los hospederos, $S$. ares muestra poca especificidad, encontrándose mayoritaria-mente en géneros de roedores de la familia Sigmodontidae (Abrothrix) (Muridae), aunque también ha sido reportada en Octodontidae (Aconaemys Ameghino y Spalacopus Wagler) y Didelphidae (marsupiales del género Marmosa Gray) durante todos los meses del año.

Hasta la fecha se han registrado 159 individuos de $S$. ares (Beaucournu \& Kelt 1990; Jameson \& Fulk 1977; Hastriter et al. 2001; Rothschild 1911 y Smit \& Rosicky 1972), de los cuales 58 han sido extraídos de A. olivaceus y 30 en A. longipilis. En este trabajo se obtuvieron 135 individuos, 70 (18 $\sigma^{\star}$ y 52 o ) de los cuáles fueron obtenidos en A. olivaceus y $65\left(210^{\star}\right.$ y 44 ㅇ ) en A. longipilis, constituyendo el mayor número de especímenes recolectados de esta especie en Chile.

Encontró a $S$. ares durante casi todos los meses del año excepto en mayo y diciembre. Nuestros resultados permiten determinar que $S$. ares es posible recolectarla en estos meses.

Hastriter et al. (2001) señalan que $S$. ares es una especie muy común, con un rango geográfico conocido en Chile que va desde la provincia de Elqui en el norte hasta el sur de la provincia de Magallanes, y al este de las provincias de Río Negro y Neuquén en la Argentina. Este mismo autor al revisar la literatura plantea que $S$. ares es posible encon-trarla durante gran parte del año, exceptuando los meses de mayo y diciembre, a lo cual de acuerdo con nuestros resultados podemos agregar que $S$. ares es posible recolectarla en estos meses.

Hastriter et al. (2001) señalan que $S$. ares es 
una especie muy común, con un rango geográfico conocido en Chile que va desde la provincia de Elqui en el norte hasta el sur de la provincia de Magallanes, y al este de las provincias de Río Negro y Neuquén en la Argentina. Este mismo autor con los datos existentes a la fecha plantea que $S$. ares es una especie presente en el ambiente gran parte del año, exceptuando los meses de mayo y diciembre, carentes de registros hasta esta investigación.

\section{AGRADECIMIENTOS}

Este trabajo forma parte de la tesis del autor en el marco del Programa de Magíster en Ciencias Mención Zoología de la Universidad de Concepción. Agradezco a la Escuela de Graduados de la Universidad de Concepción por la beca otorgada durante la permanencia en el Programa. A Andrés O. Angulo, Jorge N. Artigas, Juan C. Ortiz y Viviane Jerez R., Marcela Astorga, Patricia Berríos, Tania Olivares, Viviana Olmos, Marcela Vidal, Marcela Rodríguez y Rodrigo Moreno por su apoyo, sugerencias y críticas que hicieron posible esta investigación. Agradezco además a los correctores anónimos por sus valiosos comentarios y aportes al manuscrito.

\section{BIBLIOGRAFIA}

Alarcón, M. 2000. Estado actual del conocimiento de los Sifonápteros presentes en Chile (Insecta: siphonaptera). Gayana. 64 (1): 1-17.

Autino, A. G. \& M. Lareschi. 1998. Siphonaptera In: Biodiversidad de artrópodos argentinos. Una perspectiva biotaxonómica. Eds. Morrone \& Coscarón. Cap. 27. Ed. Sur. 279-288.

Beaucournu J. C. \& J. A. Alcover. 1989 (1990). Puces récoltées dans la province de Neuquén (Argentine); decription de 4 nouveaux taxa (Insecta: Siphonaptera). Ann. Parasit. Hum. Comp. 64: 489-505.

Beaucournu J.C., \& M. H. Gallardo. 1978. Quelques nouvelles puces du Chili (Siphonaptera) parasites de Ctenomys (Rod. Octodontidae). Bull. Soc. Path. Exot. 70: 438-450.

Beaucournu J. C., \& M. H. Gallardo. 1989. Contribution a la faune du Chili; puces nouvelles de la moitié nord (Insecta, Siphonaptera). Revue suisse Zool. 94: 181-188.

Beaucournu J. C \& M. H. Gallardo, 1991a. Catalogue provisoire des Puces du Chili. 1ére. partie (Insecta: Siphonaptera). Bull. Soc. Fr. Parasit 9 (2): $237-270$.

Beaucournu J. C \& M. H. Gallardo, 1991 b. Siphonaptères du Chili; description de 4 espèces nouvelles (Siphonaptera). Bull. Soc. Entom. France. 96: 185-203.

Beaucournu J. C. \& M. H. Gallardo, 1992. Catalogue provisoire des Puces du Chili. 2 éme partie (Insecta: Siphonaptera). Bull. Soc. Fr. Parasit. 10 (1): 93-130 .

Beaucournu J.C. \& D. A. Kelt, 1990. Contribution à la faune du Chili: puces nouvelles ou peu connues de la partie sud (Insecta, Siphonaptera). Revue Suisse Zool. 97: 647-668.

Beaucournu J. C., \& H. Launay. 1990. Les Puces (Siphonaptera) de France et du Bassin mediterranée occidental in: Faune de France. Federation Française des Science Naturelles. 14-45 .

Beaucournu J. C., \& J. C. Torres-Mura. 1986. Un Tetrapsyllus nouveau du Chili (Siphonaptera, Rhopalopsyllidae). Revue Fr. Entomol. 8: 912.

Beaucournu J.C., M. H. Gallardo \& H. Launay. 1986. Puces (Siphonaptera) nouvelles ou peu connues du Chili: description de Plocopsylla diana $\mathrm{n}$. sp. (Stephanocircidae). Ann. Parasit. Hum. Comp. 61: 359-366 .

Beaucournu J. C., J. C. Torres-Mura \& M. H. Gallardo. 1988. Description de la femelle de Ctenoparia topali Smit, 1963 et clef dicho-tomique du genre Ctenoparia Rothschild, 1909 (Siphonaptera, Hystrichopsyllidae). Ann. Parasit. Hum. Comp. 63: 380-383.

Begon, M. J.HARPER \& C. TownSEnd, 1996. Ecology individuals, populations and communities. Third Edition. Blackwell Science Ltda. 1068 pp.

Blanchard, E., 1852. Pulicidas. In: Gay, C. Historia física y política de Chile.7: 325-327.

Dunnet G. M., \& D. K. Mardon. 1974. A Monograph of Australian Fleas (Siphonaptera). Australian Journal of Zoology. 30: 273 .

Dunnet G. M., \& D. K. Mardon. 1991. Siphonaptera. In: CSIRO The insects of Australia 2: 705-716.

Glade, A (ED). 1988. Libro Rojo de los Vertebrados de Chile.A CONAF. Impresoras Comerciales S.A. Santiago. $65 \mathrm{pp}$.

Hastriter, M.W. 2001. Fleas (Siphonaptera: Ctenophthalmidae and Rhopalopsyllidae) from Argentina and Chile with two new species from the rock rat. Aconaemys fuscus. in Chile.Annals of Carnegie Museum.70(2): 169-178.

Hastriter, M., M. Alarcón \& M. Whiting. 2001. A collection of fleas (Siphonaptera) from the San Martín Reserve, Valdivia Province, Chile. Proc. Entomol. Soc. Wash. 103(2) 437-443.

Hopkins G.H.E. \& M. RothschiLD, 1956. An illustrated catalogue of the Rothschild collection of fleas (Siphonaptera) in the British Museum (Natural His- 
tory), Coptopsyllidae, Vermipsyllidea, Stephanocircidae, Ischnopsyllidae, Hypsophthalmidae and Xiphiopsyllidae. British Museum, Vol. 2: 445 pp., 1 map y 32 plates.

Hopkins G.H.E. \& M. RothschILD, 1962. An illustrated catalogue of the Rothschild collection of fleas (Siphonaptera) in the British Museum (Natural History), Hystrichopsyllidae (Acedestiinae, Anomiosyllinae, Hystrichopsyllinae, Neopsyllinae, Rhadinopsyllinae and Stenoponiinae). British Museum, Vol. 3: 559 pp ., 10 plates

Hopkins G.H.E. \& M. RothschiLd, 1966. An illustrated catalogue of the Rothschild collection of fleas (Siphonaptera) in the British Museum (Natural History), Hystrichopsyllidae (Ctenophthalminae, Dinopsyllinae, Doratopsyllinae and Listropsyllinae). British Museum, Vol. 4: 549 pp ., 12 plates.

JAMESON E.W. \& G. W. FulK, 1977. Notes on some fleas (Siphonaptera) from Chile. J. Med. Entomol. 14: 401-406.

LEwIS R.E., \& A. E. SpOTORNo. 1984. A new subespecies of Agastopsylla nylota (Siphonaptera: Hystrichopsyllidae) from Chile, with a key to the known taxa. J. Med. Entomol. 21: 392-394.

Macchiavello, A. 1948. Siphonaptera de la Costa SurOccidental de América (Primera Lista y Distribución Zoo-geográfica). Bol. Ofic. Sanit. Panamer. 27: 412-460.

Macchiavello, A. 1954. Reservoirs and vectors of plague. Journal of Tropical Medicine and Hygiene. 57: $68 \mathrm{pp}$

Mc Evedy, C.1988. La Peste Negra In: Investigación y Ciencia. 169: 82-87.

Mann, G., 1978. Los pequeños mamíferos de Chile marsupiales, quirópteros, edentados y roedores. Gayana Zool. 40: 342

Múrua, R., P. Meserve., L. González \& C. Jofré. 1987. The small mammal community of a chilean temperate rain forest: lack of evidence of competition between dominant species. Journal of Mammalogy. 68 (4): 729-738.
Muñoz-Pedreros, A. 2000. Rodentia In: Mamíferos de Chile.CEA Ediciones/A. Muñoz-Pedreros/J. Yáñez. 464 pp.

Osgood, H. 1943. The mammals of Chile Zoological series. Field Museum of Natural History. 30: $268 \mathrm{pp}$.

Pearson., O. 1983. Characteristics of a Mammalian Fauna from Forest in Patagonia, southern Argentina. J. Mamm. 64(3): 476-492

RothschiLd, N. C. 1904. Description of a new species of Siphonaptera from South America. Rev. Chil. Hist. Nat. 8: 147.

Rothschild, N. C. 1906. New species of Chilian Siphonaptera. Rev. Chil. Hist. Nat. 10: 35-39.

RothschiLd, N. C. 1909a. A new flea from Chili. Rev. Chil. Hist. Nat. 13 (1): 104-106.

RothSCHILD, N. C. 1909b. A new specie of Stephanocircus from Chile. Rev. Chil. Hist. Nat. 13(2): 181-183.

RothsChILD, N. C. 1910. New Chilian Siphonaptera. Rev. Chil. Hist. Nat. 14: 25-28.

RothsCHILD, N.C.1911. Some new genera and species of Siphonaptera. Novitates Zoologicae. 18:117-122.

Ruiz Del Río, A., 1938. Contribución a Estudio de las Enfermedades Parasitarias Humanas trans-mitidas por las Ratas en Concepción. Tesis de Grado para optar al título de Médico-Cirujano en la Universidad de Chile. 82 .

SмIT, F. G. A. M. 1955. Siphonaptera from Bariloche, Argentina, collected by Dr. J. M. de la Barrera in 1952-1954. Trans. Roy. Ent. Soc. London. 107: 319-339.

Sмiт, F. G. A. M. 1968. Siphonaptera taken in formalintraps in Chile. Zool. Anzeig. 180: 220-228.

SMIT, F. G. A. M. 1973. Siphonaptera In: Insects and other Arthropodos of Medical Importance. British $\mathrm{Mu}-$ seum (Natural History) 325-371.

Smit, F. G. A. M.\& B. Rosicky, 1972. Some Siphonaptera from Chile. Folia Parasitol. 19: 365-368.

YÁÑEZ, J., \& JAKSIC, F. 1977. Variación con la edad de algunos caracteres morfológicos de tres poblaciones de Akodon olivaceus (Rodentia: cricetidae) Bol. Mus. Nac. Hist. Nat. 35: 113-120. 